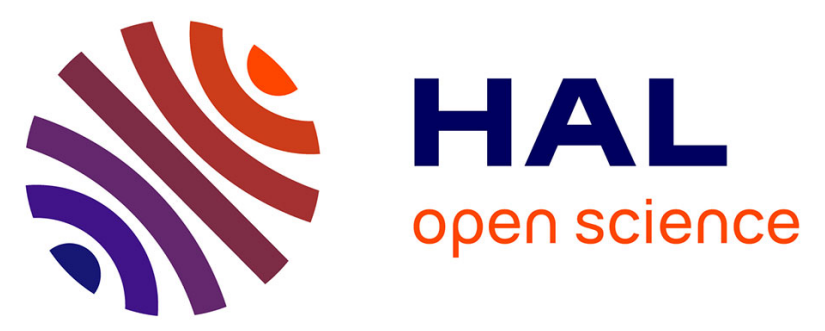

\title{
Engineering plasmonic nanostructured surfaces by pulsed laser deposition
}

Matteo Ghidelli, Luca Mascaretti, Beatrice Roberta Bricchi, Andrea Zapelli, Valeria Russo, Carlo Spartaco Casari, Andrea Li Bassi

\section{- To cite this version:}

Matteo Ghidelli, Luca Mascaretti, Beatrice Roberta Bricchi, Andrea Zapelli, Valeria Russo, et al.. Engineering plasmonic nanostructured surfaces by pulsed laser deposition. Applied Surface Science, 2018, 434, pp.1064-1073. 10.1016/j.apsusc.2017.11.025 . hal-03255117

\section{HAL Id: hal-03255117 \\ https://hal.science/hal-03255117}

Submitted on 9 Jun 2021

HAL is a multi-disciplinary open access archive for the deposit and dissemination of scientific research documents, whether they are published or not. The documents may come from teaching and research institutions in France or abroad, or from public or private research centers.
L'archive ouverte pluridisciplinaire HAL, est destinée au dépôt et à la diffusion de documents scientifiques de niveau recherche, publiés ou non, émanant des établissements d'enseignement et de recherche français ou étrangers, des laboratoires publics ou privés. 


\title{
Engineering plasmonic nanostructured surfaces by pulsed laser deposition
}

\author{
Matteo Ghidelli ${ }^{1}$, Luca Mascaretti ${ }^{1}$, Beatrice Roberta Bricchi ${ }^{1}$, Andrea Zapelli $^{1}$, Valeria Russo ${ }^{1}$, \\ Carlo Spartaco Casari ${ }^{1,2}$, Andrea Li Bassi ${ }^{1,2}$ \\ ${ }^{1}$ Micro- and Nanostructured Materials Laboratory, Department of Energy, Politecnico di Milano, via Ponzio \\ 34/3, 20133, Milano, Italy. \\ ${ }^{2}$ Center for Nanoscience and Technology - IIT@Polimi, via Giovanni Pascoli 70/3, 20133, Milano, Italy
}

\begin{abstract}
The synthesis and the optical response of gold nanoparticles (NPs) and thin nanostructured films grown by pulsed laser deposition (PLD) are here studied. Different PLD process parameters - including background gas pressure and the number of laser shots as well as post-deposition annealing treatments - have been varied to control the growth of Au NPs and films, thus tuning the surface plasmon characteristics. The mechanisms of NPs and film growth have been explored performing a morphological characterization by Scanning Electron Microscopy (SEM) and Atomic Force Microscopy (AFM), and the correlation with the optical behavior is investigated. We show that the size distribution and the morphology of the as deposited Au NPs depend on growth mechanisms which are controlled by tuning the deposition process, while the optical behavior is strongly affected by the average size and surface density of NPs or by the length of percolated Au domains. Furthermore, nucleation in gas phase has been reported at high (1000 Pa Ar) background pressures, enabling independent control of NP size and coverage, contrary to surface driven NP growth by diffusion and aggregation on substrate.
\end{abstract}

\section{Keywords}

Metallic nanoparticles; nanostructured films; pulsed laser deposition; optical behavior; surface plasmon resonance 


\section{Introduction}

Metallic nanoparticles (NPs) have been used in colored glasses since the ancient times as witnessed by the famous case of the Lycurgus cup [1]. In recent years, the potential use of metallic NPs in advanced applications has attracted the attention of the scientific community because of tunable optical properties including plasmonic effects [2]. The size confinement of metallic NPs at the nanometer scale enables the activation of the localized surface plasmon resonance (LSPR), resulting in a collective electron oscillation triggered by the selected light frequencies [3]. The LSPR wavelength depends on the NPs size distribution, density, shape as well as on the surrounding dielectric medium [2-4]. Nanoengineered NPs enable to tune specific LSPR wavelengths as requested by several applications in the fields of optoelectronics [2], sensors [2, 5], catalysis [6, 7], nanomedicine $[8,9]$ as well as substrate development for Surface Enhanced Raman Scattering (SERS) $[10,11]$. Furthermore, the integration of plasmonic NPs within wide band gap semiconductor oxides (such as $\mathrm{TiO}_{2}$ and $\mathrm{ZnO}$ ) is a promising field as well, with the possibility to improve photoelectrocatalytic performances by minimizing electron/hole recombination, while increasing visible light absorption $[2,6,12]$. Here, applications span in the field of thin film photovoltaics $[2,13]$, solar fuel production with hydrogen generation (solar water splitting) [14] as well as photocatalysis [6].

Metallic NPs are often produced by liquid phase chemical techniques involving nucleation and growth phenomena [15]. These techniques enable the possibility to produce different metallic NPs (Au, Ag, Cu etc.) with a good control of the size distribution and shape $[2,15]$. NPs produced by liquid phase have been integrated in wide band gap semiconductors [16]. For instance, Au and Ag NPs were successfully infiltrated in $\mathrm{TiO}_{2}$ thin films by Valverde et al. [16] and Qi et al. [17] in order to improve the photoconductivity of thin film photovoltaic devices (i.e. Dye Sensitized Solar Cells, DSSCs). Similarly, Chen et al. [18] infiltrated chemically produced Au NPs within vertically aligned ZnO nanorods again for photovoltaic applications. However, a main drawback of this methodology is represented by the use of aggressive solvents [15], which can induce damaging especially for delicate applications involving polymeric substrates [2]. In addition, a two-step procedure - namely the synthesis of thin film followed by the NPs infiltration - combined with the presence of 2 
contaminants (i.e. the reaction products) represents a further limitation of this technique $[2,13,16,17]$ in terms of integration efficacy and available morphologies.

Surface supported metallic NPs have been synthetized by vapor phase techniques involving thermal evaporation $[7,19]$, electron beam evaporation [12], reactive sputtering [20] as well as atomic layer deposition [21]. The advantages are represented by the possibility to produce highly pure NPs without the presence of chemicals, while providing a good control of the size distribution resulting in a fine tuning of the optical behavior $[2,19]$. For these reasons, vapor phase produced NPs have been integrated within wide band gap semiconductors, especially $\mathrm{TiO}_{2}[7,12]$. Awazu et al. [7] and Mubeen et al. [12] integrated thermal evaporated $\mathrm{Ag}$ and $\mathrm{Au} \mathrm{NPs}$ in $\mathrm{TiO}_{2}$, respectively, to improve the photocatalytic behavior and for hydrogen production using solar energy. Nevertheless, the integration of metallic NPs produced by vapor phase within semiconductors is often limited by surface diffusion or by the presence of a heating step, especially for thermal and electron beam evaporation when NPs are produced by dewetting of continuous thin $(<10 \mathrm{~nm})$ films [7, 12, 19]. Moreover, sputtering and atomic layer deposition do not enable a sufficient flexibility to tune the NPs size and shape distribution, thus reducing the application range in terms of the optical behavior [20,21].

In comparison to the aforementioned techniques, pulsed laser deposition (PLD) is known for high deposition rate with high kinetic energy species resulting in a promising approach to produce either NPs or continuous metallic and oxide films [22-26]. The former studies belong to Gonzalo et al. [22], Donnelly et al. [23] and Domingo et al. [24] with the deposition of small $(<5 \mathrm{~nm})$ Au NPs as SERS substrates, without investigating the effect of the numerous PLD parameters. Similarly, Agarwal et al. deposited Au NPs by PLD for SERS applications [27] and investigated the growth mechanism of PLD deposited Au thin films [10]. The integration of PLD deposited Au NPs - with a controlled size distribution - within thin oxide films has been successfully carried out by Orlianges et al. [28] and Resta et al. [29], respectively in $\mathrm{VO}_{2}$ and $\mathrm{TiO}_{2}$, while Konda et al. [30] deposited Au NPs at the interface of $n-C d S e / p-S i$ heterojunction diodes to improve their optical absorption. The study of the effect of PLD parameters has been carried out by Alonso et al. [31] evaluating the plasmonic response as a function of the laser shot number which influences the average size of Ag NPs as well 
as the formation of percolating structures before the film growth. Verma et al. investigated the optical behavior of NPs produced by PLD by varying the substrate heating [32], the target-to-substrate distance [33], the laser fluence [33] as well as changing the composition, i.e. studying Ag-Au NPs [34] and they reported the formation of large NPs (diameter $>50 \mathrm{~nm}$ ) only if the substrate is heated at $\mathrm{T} \geq 150{ }^{\circ} \mathrm{C}$ during deposition. Finally, by exploiting in-plume cluster nucleation phenomena at high pressures, PLD offers the possibility to deposit NPs whose size distribution is independent of the substrate, with clear advantages when dealing with multiple substrate depositions [35].

Nevertheless, a complete study related to the effect of PLD key process parameters involving the number of laser shots and background gas pressure together with a thorough structural and optical characterization has not been performed yet, while in-plume cluster nucleation of metallic NPs has barely explored from experimental point of view for plasmonic applications. Furthermore, the influence of PLD process parameters on film growth mechanisms and on the structural and optical properties still demands a detailed comprehension. The understanding of these phenomena is fundamental to develop as-deposited devices based on metallic nanostructures (i.e. NPs and thin films) exhibiting the desired morphological and optical properties, as opposed to the most common PVD approach involving growth of a continuous film and a post-annealing dewetting process.

In this work, we deposited Au NPs by PLD varying a wide range of process parameters as well as exploiting post-deposition annealing treatments of percolated nanostructured films. A complete structural characterization involving Scanning Electron Microscopy (SEM) and Atomic Force Microscopy (AFM) has been carried out and related with the transmittance optical behavior. We show that: (i) the size distribution and the morphology of the as deposited Au NPs strongly depend on the number of shots and the background gas pressure and we show that Au NPs up to $16 \mathrm{~nm}$-diameter can be produced without substrate heating or annealing treatments; (ii) the optical behavior is strongly affected by the average size of NPs, the average length of percolated Au domains as well as by the morphological changes induced by the post-deposition thermal annealing treatment; (iii) in-plume nucleation phenomena occur at relatively high (1000 Pa) 4 
background pressures; (iv) annealing treatments provide the formation of larger $(23 \mathrm{~nm}) \mathrm{NPs}$. Finally, the mechanisms of film growth as a function of PLD parameters involving the formation of NPs as well as of percolating and continuous films are explored.

\section{Experimental}

Au NPs and films have been deposited by ablating an Au (99.99\%) target with a ns-pulsed laser (Nd:YAG, 2nd harmonic, $\lambda=532 \mathrm{~nm}$, repetition rate $1 \mathrm{~Hz}$, pulse duration $5-7 \mathrm{~ns})$. The laser fluence on the target was set at about $2.0 \mathrm{~J} / \mathrm{cm}^{2}$ and the laser pulse energy was $100 \mathrm{~mJ}$. Si (100) and soda-lime glass were used as substrates after delicate cleaning in ultrasonic bath with isopropanol. The substrates have been mounted on a rotating sample holder at a fixed target-to-substrate distance $\left(d_{t-s}\right)$ of $50 \mathrm{~mm}$. All the depositions have been carried out at room temperature within a pure Ar background gas, varying the pressures (from 10 up to $1000 \mathrm{~Pa}$ ) as well as the number of shots (from 50 up to 1000) to explore different coverages and thus the film growth mechanisms and the subsequent change of the optical behavior. For the $1000 \mathrm{~Pa}$ pressure, samples have been produced with 75 and 250 shots as well, to explore in-plume cluster nucleation. Post-deposition annealing treatments were performed in air in a Lenton muffle furnace with $4^{\circ} \mathrm{C} / \mathrm{min}$ heating ramp and $2 \mathrm{~h}$ dwell at $500^{\circ} \mathrm{C}$ and $650^{\circ} \mathrm{C}$. The morphological characterization was carried out using a field emission scanning electron microscope (FEGSEM, Zeiss Supra 40) for NPs and films deposited on Si (100) substrate. The obtained micrographs were analyzed by Image J software to extract the size distribution and the coverage. Furthermore, selected samples have been investigated by atomic force microscope (AFM, Thermomicroscope Autoprobe CP Research) imaging in tapping mode using TESP-SS tips with a $2 \mathrm{~nm}$ curvature radius. Optical transmittance spectra (in the range 250 - $2000 \mathrm{~nm}$ ) were evaluated with a UV-vis-NIR PerkinElmer Lambda 1050 spectrophotometer with a 150 $\mathrm{mm}$ diameter integrating sphere. All the acquired spectra were normalized with respect to glass substrate contribution by setting equal to 1 the intensity at the glass/Au interface.

\section{Results and discussion}




\subsection{Morphology of as deposited Au NPs and films}

Figure 1 is a collection of SEM images displayed as a function of the background gas (Ar) pressure and the number of laser shots. Increasing the number of shots at fixed $10 \mathrm{~Pa}$ pressure, we observed that the morphology changes from NPs (50 and 100 shots) to extended percolating domains (500 shots), up to a continuous film in which crystalline grains are clearly visible (1000 shots). As reported by Alonso et al. [31] and Agarwal et al. [10], the increment of the number of shots induces the deposition of a larger material quantity (i.e. increment of the coverage) favoring the extension of crystalline domains and leading to film formation. Conversely, if the shot number increases at larger pressures (500 and $1000 \mathrm{~Pa}$ ), a continuous film is not formed even at 1000 shots, rather showing long percolating domains (see also Figure $2 \mathrm{~b}$ ). This is attributed to the lower kinetic energy of the ablated species as well as to the larger plume confinement, which induce lateral dispersion of the species and smaller deposition rate at the substrate (as well as gas phase cluster nucleation, see below). Furthermore, evidence of the growth of a second NPs layer is visible at 1000 shots as the presence of brighter zones on top of the percolating domains (see the magnified Figure $2 \mathrm{~b}$ ). On the whole, for our experimental conditions, we found that NPs are formed only at 50 and 100 shots regardless of the pressure range, while long percolating domains or films are deposited at 500 and 1000 shots (Figure 1 and Figure 2). An increment of the background pressure - at fixed shot number - prevents the formation of a continuous film, leading to shorter percolating domains, while influencing the average size of NPs. As a matter of fact, it can be observed that the largest ( $16 \mathrm{~nm}$ diameter) NPs are obtained at 100 shots, $100 \mathrm{~Pa}$ (see also Figure $3 \mathrm{~b}$ ). NPs have a rounded shape in agreement with most of the literature dealing with PLD deposited NPs $[10,27,33,36]$. A similar shape is found for other NPs (Figure 1 at 50 and 100 shots). A Gaussian distribution profile of diameters has been observed for all NPs (an example is reported in Figure 3a for the $10 \mathrm{~Pa}, 100$ shots) in agreement with literature results by Verma et al. [33]. Specifically, NPs deposited at $10 \mathrm{~Pa}, 100$ shots have an average diameter equal to $9 \mathrm{~nm}$ with a maximum of $16 \mathrm{~nm}$ (Figure 3a). 
Figure $3 \mathrm{~b}$ shows the evolution of the Au NPs diameter as a function of the background pressure for 50 and 100 shots, respectively. The NPs size reaches a maximum at a pressure equal to $100 \mathrm{~Pa}$. For this condition, in plume cluster nucleation phenomena combined with a large kinetic energy when landing on the substrate induce the formation of larger NPs (large surface diffusion). At higher pressures, the average NPs size decreases because of the formation of a less directional ablation plume, leading to higher dispersion within the deposition chamber. On the other hand, at lower pressures (i.e. $10 \mathrm{~Pa}$ ) in plume cluster nucleation phenomena are reduced promoting growth phenomena only by surface diffusion and coarsening $[37,38]$. Hence, NPs growth is controlled by two different mechanisms, namely in-plume cluster nucleation and surface diffusion, which respectively dominate at high $(>300 \mathrm{~Pa})$ and low $(\leq 300 \mathrm{~Pa})$ pressures. Increasing the shot number from 50 up to 100 induces an increase of the average NPs size because of the larger amount of deposited material (surface coalescence). However, at large pressures (500 and $1000 \mathrm{~Pa}$ ) both trends converge toward an average size of $\sim 6 \mathrm{~nm}$. Here, in plume clustering effects prevail over the substrate diffusion. Figure $3 c$ shows the coverage as a function of the number of shots. The coverage increases when increasing the number of shots as visible also in Figure 1 in which the morphology evolves from NPs toward a continuous film. When increasing the pressure at fixed shot number, the coverage is reduced because of the large lateral dispersion, see Fig. 1.

AFM measurements have been performed to estimate the height of selected NPs and to provide information about their aspect ratio. Figure 4 shows the NPs formed at 50 and 100 shots at $1000 \mathrm{~Pa}$, reporting an average height of $\sim 6 \mathrm{~nm}$. This indicates that at this pressure NPs have an almost spherical shape since the diameter, measured by SEM, is equal to $\sim 4 \mathrm{~nm}$ (Figure 3b). Furthermore, AFM images report an increment of the NP coverage as function of the number of shots (as in Figure 3c), while showing that the average height is independent on the number of shots, in agreement with in-plume cluster nucleation phenomena at high pressures, as discussed more in detail below (Section 3.2c). For a different growth regime (100 Pa) and 100 shots, AFM analyses reveal the formation of NPs with irregular shape as provided by surface aggregation phenomena (Figure 1), whose average height is around 7-8 nm, indicating flattening occurring at low pressures. 


\subsection{Optical properties}

This section is organized focusing first on the effect of the shot number at fixed pressure and on the background pressure at fixed shot number; then, in-plume cluster nucleation phenomena are explored, providing a complete understanding of the optical behavior.

\section{a) Effect of the number of shots at fixed pressure}

The study of the surface plasmon resonance (SPR) characteristics of Au NPs and films has been carried out by performing optical transmission measurements. Figure 5a-b shows the transmission spectra as a function of the number of shots for two different growth regimes, respectively $10 \mathrm{~Pa}$ and $1000 \mathrm{~Pa}$. At $10 \mathrm{~Pa}$ pressure, the optical properties abruptly change depending on the presence of NPs (50 and 100 shots) or a film (500 and 1000 shots), Figure 5a. Specifically, when NPs are formed the optical behavior clearly shows the presence of a SPR peak (the position of the transmittance minimum was determined by Gaussian fitting) and located in the visible range at $562 \mathrm{~nm}$ and $606 \mathrm{~nm}$, respectively for 50 and 100 shots. The redshift of the SPR wavelength is related to the increment of NPs size, which changes from $5 \mathrm{~nm}$ to $10 \mathrm{~nm}$ on average (Figure $3 \mathrm{~b}$ ), in agreement with the literature $[31,33]$. Furthermore, the reduction of the average transmittance in the visible range upon increasing the number of shots can be related with an increase of the coverage, Figure 3c. On the other hand, the transmission spectra of continuous films produced at 500 and 1000 shots do not show any distinct dip in the visible range, as for NPs, while displaying a transmittance peak located at around 560 and $520 \mathrm{~nm}$, respectively. This kind of optical response is related to the development of continuous or percolating films, as shown in Figure 1. Specifically, the observed decreased transmission at wavelengths larger than $500 \mathrm{~nm}$ is attributed to free electrons response of the Au films promoting absorption in the IR [39] as well as by the large ( $80 \%)$ reflectance value for continuous film obtained at 1000 shots (Figure 5a). On the other hand, at lower wavelengths $(<500 \mathrm{~nm})$ the decrement of transmission is attributed to bound electrons response or inter-band transitions [39], as proven by the relatively low reflectance values $(<20 \%)$. Furthermore, it can be noted that the 1000 shots film exhibits a lower transmittance as provided by the larger coverage, Figure 2c. At $1000 \mathrm{~Pa}$ there is no formation of a continuous film or with extended percolated domains even at large shot number 8 
(Figure 1), for this reason the transmission spectra reported in Figure $5 b$ show a plasmonic peak in the visible range. The redshift observed upon increasing the number of shots is related to the progressive formation of longer percolating structures without leading to a continuous film, Figure 1 and Ref. [31]. Specifically, it can be observed that the surface plasmon resonance wavelength $\left(\lambda_{S P R}\right)$ linearly increases from 540 to $685 \mathrm{~nm}$, when increasing the size of the NPs and percolating domains (Figure 5c), while an almost negligible $(<10 \%)$ reflectance is observed even for the $1000 \mathrm{~Pa}, 1000$ shots percolated film. The SPR broadening was determined from full width at half maximum (FWHM) in the transmission dip with reference to the transmission at interband edge near $500 \mathrm{~nm}$ (see inset of Figure $5 \mathrm{~b}$ ). This enables to determine the SPR response by avoiding the change in overall transmission due to different coverage of the films. In Figure $5 b$ it can be qualitatively observed that the full width at half maximum (FWHM) increases at larger number of shots, being possibly connected with the larger size dispersion occurred in the percolating domains (Figures 1 and 3c). Finally, Figure $5 c$ shows that the optical transmittance decreases from $97 \%$ down to $68 \%$ because of the larger coverage (Figure 3c).

\section{b) Effect of the background gas pressure at fixed number of shots}

Figure 6 shows the evolution of optical transmittance as a function of the pressure for a fixed shot number equal to 50, 100 and 500 shots, respectively in Fig.6a, b and c. When Au NPs are formed (namely low number of shots, Figure $6 a-b)$, the pressure plays a role on the control of the optical transmittance as well as on the position of the $\lambda_{S P R}$. Specifically, we found that the pressure affects the average NPs size distribution as reported in Figure 3b and discussed above. Decreasing the pressure, the NPs size is reduced leading to a blue shift (decrement) of the $\lambda_{\text {SPR }}$. However, a maximum size is found in correspondence of the 100 Pa deposited specimens in which we have shown the formation of larger NPs. Figure $6 c$ shows the evolution of the optical properties as a function of the pressure at fixed 500 shots; from Figure 1, we can observe in this case the formation of percolating domains whose average size increases when decreasing the pressure down to $10 \mathrm{~Pa}$ where an almost continuous film is formed. Optical properties evolve as well, specifically they change from NP- 
like $(1000 \mathrm{~Pa})$ showing the presence of a SPR dip toward a progressive reduction of the transmission in the infrared range combined with the enhancement of a transmittance peak around $500 \mathrm{~nm}$. This behavior is related with the formation of even longer percolating domains, see Ref. [39]. At 10 Pa the film is almost continuous (Figure 1), thus reporting a bulk metallic behavior with the absence of a SPR and enhanced absorption/reflection above and below the transmission peak, see Section 3.2a and Refs. [33, 39]. Furthermore, it can be observed that the broadening effects are enhanced when decreasing the pressure from $1000 \mathrm{~Pa}$ down to $100 \mathrm{~Pa}$. This is related with the larger variability in size and shape of the percolating domains.

The above observations highlight the empirical effect of varying the PLD process parameters on the optical properties and can be practical utility; however, in order to find a correlation with the intrinsic NPs properties, the main optical properties have been plotted as a function of the NPs average diameter obtained at different pressures (Figure 7). Figure 7a shows (for a fixed number of shots, i.e. 100) that the $\lambda_{S P R}$ linearly increases for large diameter NPs from 540 up to $630 \mathrm{~nm}$, see also Refs. [33, 36]. Furthermore, an SPR broadening effect occurs when changing the pressure, Figure 6a,b. A quantitative analysis carried out from Figure $6 \mathrm{~b}$ and reported in Figure 7 shows that SPR broadening increased monotonically with average NPS diameter evolving from of 100 up to $200 \mathrm{~nm}$ [33]. As it can be qualitatively observed in Figure 1, the diameter size and shape dispersion (which has a maximum at $100 \mathrm{~Pa}$ ) mainly contribute to the overall broadening as also reported in Ref. [31]. Other than the SPR wavelength and its broadening, the transmittance dip evolves as a function of the pressure, Figures $7 b$ and $7 c$. Specifically, it can be observed that in all the cases transmittance decreases for the larger diameters which, at the fixed number of shots, correspond to the larger amount of deposited materials and to the larger coverage (Figure 3c). This has been also observed in Figure $5 c$ increasing the shot number at fixed pressure.

Finally, Figure 8 shows the evolution of the $\lambda_{S P R}$ as a function of coverage for percolated films showing a increment from $610 \mathrm{~nm}$ up to $760 \mathrm{~nm}$. This trend is related with the increased dimension of the percolated domains obtained at lowest pressures (Figure 1). 


\section{c) In-plume cluster nucleation phenomena}

The effect of the background gas pressure at large pressures has been investigated in order to explore in-plume cluster generation phenomena. Specifically, the number of shots has been finely varied from 50 up to 250 at $1000 \mathrm{~Pa}$, and then an analysis of the average size of NPs, coverage and optical transmittance has been carried out. Figure 9a shows the evolution of the average diameter and coverage as a function of the number of shots. It can be observed that upon increasing the number of shots (up to 250, i.e. before occurrence of coalescence or formation of percolated structures, see Fig.1) the average diameter of NPs is almost constant and around 4$5 \mathrm{~nm}$, while the coverage increases from 8 up to $27 \%$. The optical behavior shows that the $\lambda_{S P R}$ does not vary with the number of shots being constant and equal to $542 \mathrm{~nm}$ (Figure 9b). These results prove the in-plume cluster nucleation phenomena at large pressure, while showing negligible NPs growth by surface diffusion and coarsening on the substrate. Specifically, the large background gas confinement coupled with the small kinetic energy of the ablated species induces the formation of in-plume clusters, with reduced substrate mobility, thus inducing the deposition of NPs with constant average size (Figure 9a). On the other hand, the coverage increases with increasing number of shots, affecting the optical transmittance at $\lambda_{S P R}$ which decreases form 99 \% down to $92 \%$ for 50 and 250 shots, respectively.

\subsection{Effect of the annealing treatment}

Post-deposition annealing treatments of percolating films have been carried out in order to evaluate the formation of Au NPs, with the aim to obtain larger NP sizes. In the literature, similar approaches have been used for evaporated films exploiting dewetting phenomena to form of NPs starting from a few nm-thick films, Refs. [40-42]. Figure 10a is a SEM image of a $1000 \mathrm{~Pa}, 1000$ shots as deposited film in which long percolating domains can be observed covering the entire surface. After an annealing treatment at $500{ }^{\circ} \mathrm{C}$ and $650{ }^{\circ} \mathrm{C}$ in air (Figure $10 \mathrm{~b}, \mathrm{c}$ ) separated NPs with irregular shape are formed with an average lateral size of about $23 \mathrm{~nm}$, reaching even $70 \mathrm{~nm}$ as a maximum diameter. Hence, we show that annealing treatment favors the formation 
of larger NPs, providing a possible way to expand the applications of deposited films. AFM investigations carried out for the $500{ }^{\circ} \mathrm{C}$ annealed specimen (Figure 10d-e) show the presence of separated NPs with an average height of $\sim 20 \mathrm{~nm}$, providing an aspect ratio (equivalent diameter/height) almost equal to 1 , in agreement with literature studies for evaporated films after thermal treatment, Refs. [40-42].

Figure 11a shows the evolution of the coverage and the NPs average diameter as a function of the annealing temperature. The diameter slightly increases from $23 \mathrm{~nm}$ up to $30 \mathrm{~nm}$, increasing the annealing temperature from $500{ }^{\circ} \mathrm{C}$ up to $800{ }^{\circ} \mathrm{C}$. This suggests that the control of the NPs size mainly depends on the quantity of the deposited material on the substrate rather than on the annealing temperature. To verify this claim annealing treatments (at $500{ }^{\circ} \mathrm{C}$ ) have been performed on two different percolating films, namely $500 \mathrm{~Pa}$, 1000 shots and $1000 \mathrm{~Pa}, 500$ shots (not shown) reporting a different coverage and average length of percolated domains (see Figure 3c). In the former case, the average NPs diameter was $38 \mathrm{~nm}$ (with a maximum of $100 \mathrm{~nm}$ ), while in the latter case it was $20 \mathrm{~nm}$. As a matter of facts, the larger coverage (i.e. quantity of deposited material) at $500 \mathrm{~Pa}, 1000$ shots provides the formation of larger NPs after annealing. Finally, as expected (Figure 10), the coverage decreases from as-deposited percolated film to NPs because of the increase of the height combined with a larger inter-particle distance (Figure 10b,c).

Figure 11b shows the comparison of optical properties between the percolated as-deposited films and the NPs produced by annealing of a $1000 \mathrm{~Pa}, 1000$ shots film (Figure 10a). While the percolating film has a broad dip due to its long and irregular percolating domains (Section 3.2), the NPs exhibit a sharper dip located at $536 \mathrm{~nm}$ with no significant difference with respect to the annealing temperature. The SPR peak narrowing when moving from the percolated as deposited film to NPs can be related to the sharper size distribution of the surface nanostructures after annealing, Ref. [40]. Furthermore, form Figure 11b it can be observed that the NPs have an almost $100 \%$ transmittance in the infrared region, which is not the case for the percolating film whose behavior starts to resemble that of the continuous films (Section 3.2). 
The $\lambda_{\text {SPR }}$ for NPs produced by annealing can be compared with the as deposited ones (Figure 7a). Here, it can be observed that annealed NPs, with size $\sim 25 \mathrm{~nm}$, have SPR wavelengths comparable with $5 \mathrm{~nm}$ diameter as-deposited NPs (Figure 7a). This blue shift (and narrowing) of the absorption band is probably related with the change in the inter-particle distance as reported in Ref. [40], while possible other effects including the NPs shape and the aspect ratio which can play a role as well $[3,41]$. The short inter-particle distance in the asdeposited sample favors the dipolar interaction between adjacent particles which is known to widen and redshift the SPR [42]. Annealing treatment at 500 or $650{ }^{\circ} \mathrm{C}$ leads to more distant particles, characterized by a SPR band similar to that expected for isolated Au nanoparticles with clear effects on the optical behavior [42].

\section{Conclusions}

Au NPs and films have been deposited by pulsed laser deposition by varying a number of deposition parameters as well as exploiting annealing treatments, leading to the following set of conclusions:

- The size distribution and the morphology of the as deposited Au NPs depend on the number of ablation shots and the deposition background gas pressure. Specifically, we show that the coverage increases by increasing the number of shots eventually leading to the formation of a percolating or continuous films, while pressure affects the size of NPs reaching a maximum at $100 \mathrm{~Pa}$.

- Different optical behaviors are found for NPs, percolated domains and continuous films. In the case of NPs we show that the optical properties depend on the average diameter enabling to tune the surface plasmon resonance (SPR). Specifically, we show that there is an almost linear dependence between the average size of NPs and the SPR wavelength.

- In-plume nucleation phenomena have been reported at high (1000 Pa) background pressures, enabling independent control of NP size and coverage, and thus of NP size and density, contrary to NP growth by diffusion and surface aggregation occurring at lower pressures. 
- Annealing treatments have been exploited to obtain larger NPs with a relatively low number density and coverage (and thus larger inter-particle distance). We show that the average NPs size is controlled by the quantity of deposited materials, and up to $\sim 40 \mathrm{~nm}$ NPs have been obtained.

- PLD is a feasible and versatile technique to produce nanostructured surfaces with tailored optical properties. These systems can be exploited for plasmonic applications in a wide range of potential areas including SERS detection, biosensors, nano-antennas and light management.

\section{Acknowledgements}

We acknowledge Tarek Afifi Afifi for his aid in performing experiments.

\section{References}

[1] D. Barber, I.C. Freestone, An investigation of the origin of the colour of the Lycurgus Cup by analytical transmission electron microscopy, Archaeometry 32 (1990) 33-45.

[2] H.A. Atwater, A. Polman, Plasmonics for improved photovoltaic devices, Nature Materilas 9 (2000) 2052013.

[3] K.L. Kelly, E. Coronado, L.L. Zhao, G.C. Schatz, The Optical Properties of Metal Nanoparticles: The Influence of Size, Shape, and Dielectric Environment, J. Phys. Chem. B 107 (2002) 668-677.

[4] S. Grachev, M.D. Grazia, E. Barthel, E. Søndergård, R. Lazzari, Real-time monitoring of nanoparticle film growth at high deposition rate with optical spectroscopy of plasmon resonances, J. Phys. Chem. D 46 (2013) 375305.

[5] P.K. Jain, X. Huang, I.H. El-Sayed, M.A. El-Sayed, Noble Metals on the Nanoscale: Optical and Photothermal Properties and Some Applications in Imaging, Sensing, Biology, and Medicine, Accounts Chem. Res. 41 (2007) 1578-1585. 
[6] C. Fu, M. Li, H. Li, C. Li, X.G. Wu, B. Yang, Fabrication of Au nanoparticle/TiO2 hybrid films for photoelectrocatalytic degradation of methyl orange, J. Alloy Compd. 692 (2017) 727-733.

[7] K. Awazu, M. Fujimaki, C. Rockstuhl, J. Tominaga, H. Murakami, Y. Ohki, et al., A Plasmonic Photocatalyst Consisting of Silver Nanoparticles Embedded in Titanium Dioxide, Journal of American Chemical Society 130 (2008) 1676-1680.

[8] X. Huang, M.A. El-Sayed, Gold nanoparticles: Optical properties and implementations in cancer diagnosis and photothermal therapy, J. Adv, Res. 1 (2010) 13-28.

[9] N.G. Khlebtsov, L.A. Dykman, Optical properties and biomedical applications of plasmonic nanoparticles, J. Quant. Spectrosc. 111 (2010) 1-35.

[10] N.R. Agarwal, F. Neri, S. Trusso, P.M. Ossi, Growth Analysis of Pulsed Laser Ablated Films, Plasmonics 8 (2013) 1707-1712.

[11] K. Kamakshi, K.C. Sekhar, A. Almeida, J. Agostinho Moreira, M.J.M. Gomes, Tuning the surface plasmon resonance and surface-enhanced Raman scattering of pulsed laser deposited silver nanoparticle films by ambience and deposition temperature, J. Optics 16 (2014) 055002.

[12] S. Mubeen, G. Hernandez-Sosa, D. Moses, J. Lee, M. Moskovits, Plasmonic photosensitization of a wide band gap semiconductor: converting plasmons to charge carriers, Nano letters 11 (2011) 5548-5552.

[13] S.D. Standridge, G.C. Schatz, J.T. Hupp, Toward Plasmonic Solar Cells: Protection of Silver Nanoparticles via Atomic Layer Deposition of TiO2, Langmuir 25 (2009) 2596-2600.

[14] S.C. Warren, E. Thimsen, Plasmonic solar water splitting, Energy Environ. Sci. 5 (2012) 5133-5146.

[15] B.L. Cushing, V.L. Kolesnichenko, C.J. O'connor, Recent advances in the liquid-phase syntheses of inorganic nanoparticles, Chemical Reviews 104 (2004) 3893-3946.

[16] G. Valverde-Aguilar, J.A. García-Macedo, V. Rentería-Tapia, M. Aguilar-Franco, Photoconductivity studies on amorphous and crystalline TiO2 films doped with gold nanoparticles, Applied Physics A 103 (2011) 659-663. [17] J. Qi, X. Dang, P.T. Hammond, A.M. Belcher, Highly Efficient Plasmon-Enhanced Dye-Sensitized Solar Cells through Metal@Oxide Core-Shell Nanostructure, ACSNano 5 (2011) 7108-7116. 
[18] Z.H. Chen, Y.B. Tang, C.P. Liu, Y.H. Leung, G.D. Yuan, L.M. Chen, et al., Vertically Aligned ZnO Nanorod Arrays Sentisized with Gold Nanoparticles for Schottky Barrier Photovoltaic Cells, Journal of Phys. Chem. C 113 (2009) 13433-13437.

[19] N. Venugopal, A. Mitra, Influence of temperature dependent morphology on localized surface plasmon resonance in ultra-thin silver island films, Appl. Surf. Sci. 285 (2013) 357-372.

[20] P. Sangpour, O. Akhavan, A. Moshfegh, M. Roozbehi, Formation of gold nanoparticles in heat-treated reactive co-sputtered Au-SiO 2 thin films, Appl. Surf. Sci. 254 (2007) 286-290.

[21] J. Lu, J.W. Elam, P.C. Stair, Synthesis and stabilization of supported metal catalysts by atomic layer deposition, Accounts Chem. Res. 46 (2013) 1806-1815.

[22] J. Gonzalo, A. Perea, D. Babonneau, C.N. Afonso, N. Beer, J.P. Barnes, et al., Competing processes during the production of metal nanoparticles by pulsed laser deposition, Physical Review B 71 (2005).

[23] T. Donnelly, S. Krishnamurthy, K. Carney, N. Mcevoy, J.G. Lunney, Pulsed laser deposition of nanoparticle films of Au, Appl. Surf. Sci. 254 (2007) 1303-1306.

[24] C. Domingo, V. Resta, S. Sanchez-Cortes, J.V. Garcıa-Ramos, J. Gonzalo, Pulsed Laser Deposited Au Nanoparticles as Substrates for Surface-Enhanced Vibrational Spectroscopy, J. Phys. Chem. C 111 (2007) 81498152.

[25] P. Gondoni, M. Ghidelli, F. Di Fonzo, A. Li Bassi, C.S. Casari, Fabrication of nano-engineered transparent conducting oxides by pulsed laser deposition, JOVE-J. Vis. Exp. 72 (2013).

[26] P. Gondoni, M. Ghidelli, F. Di Fonzo, M. Carminati, V. Russo, A. Li Bassi, et al., Structure-dependent optical and electrical transport properties of nanostructured Al-doped ZnO, Nanotechnology 23 (2012).

[27] N.R. Agarwal, F. Neri, S. Trusso, A. Lucotti, P.M. Ossi, Au nanoparticle arrays produced by Pulsed Laser Deposition for Surface Enhanced Raman Spectroscopy, Appl. Surf. Sci. 258 (2012) 9148-9152.

[28] J.C. Orlianges, J. Leroy, A. Crunteanu, R. Mayet, P. Carles, C. Champeaux, Electrical and optical properties of vanadium dioxide containing gold nanoparticles deposited by pulsed laser deposition, Appl. Phys. Lett. 101 (2012) 133102. 
[29] V. Resta, A.P. Caricato, A. Loiudice, A. Rizzo, G. Gigli, A. Taurino, et al., Pulsed laser deposition of a dense and uniform Au nanoparticles layer for surface plasmon enhanced efficiency hybrid solar cells, J. Nanopart. Res. 15 (2013).

[30] R.B. Konda, R. Mundle, H. Mustafa, O. Bamiduro, A.K. Pradhan, U.N. Roy, et al., Surface plasmon excitation via Au nanoparticles in n-CdSe/p-Si heterojunction diodes, Appl. Phys. Lett. 91 (2007) 191111.

[31] J.C. Alonso, R. Diamant, P. Castillo, M.C. Acosta-García, N. Batina, E. Haro-Poniatowski, Thin films of silver nanoparticles deposited in vacuum by pulsed laser ablation using a YAG:Nd laser, Appl. Surf. Sci. 255 (2009) 4933-4937.

[32] S. Verma, B.T. Rao, S. Bhartiya, V. Sathe, L.M. Kukreja, Growth temperature dependent surface plasmon resonances of densely packed gold nanoparticles' films and their role in surface enhanced Raman scattering of Rhodamine6G, Appl. Surf. Sci. 346 (2015) 379-387.

[33] S. Verma, B. Tirumala Rao, S. Rai, V. Ganesan, L.M. Kukreja, Influence of process parameters on surface plasmon resonance characteristics of densely packed gold nanoparticle films grown by pulsed laser deposition, Appl. Surf. Sci. 258 (2012) 4898-4905.

[34] S. Verma, B.T. Rao, A.P. Detty, V. Ganesan, D.M. Phase, S.K. Rai, et al., Surface plasmon resonances of AgAu alloy nanoparticle films grown by sequential pulsed laser deposition at different compositions and temperatures, J. Appl. Phys. 117 (2015) 133105.

[35] R. Wood, J. Leboeuf, K.-R. Chen, D. Geohegan, A. Puretzky, Dynamics of plume propagation, splitting, and nanoparticle formation during pulsed-laser ablation, Appl. Surf. Sci. 127 (1998) 151-158.

[36] L.M. Kukreja, S. Verma, D.A. Pathrose, B.T. Rao, Pulsed laser deposition of plasmonic-metal nanostructures, J. Phys. Chem. D 47 (2014) 034015.

[37] D. Cattaneo, S. Foglio, C. Casari, A.L. Bassi, M. Passoni, C. Bottani, Different W cluster deposition regimes in pulsed laser ablation observed by in situ scanning tunneling microscopy, Surf. Sci. 601 (2007) 1892-1897. 
[38] C. Casari, S. Foglio, M. Passoni, F. Siviero, C. Bottani, A.L. Bassi, Energetic regimes and growth mechanisms of pulsed laser deposited Pd clusters on Au (111) investigated by in situ Scanning Tunneling Microscopy, Phys. Rev. B 84 (2011) 155441.

[39] Z.K. Zhou, X.N. Peng, Z.J. Yang, Z.S. Zhang, M. Li, X.R. Su, et al., Tuning gold nanorod-nanoparticle hybrids into plasmonic Fano resonance for dramatically enhanced light emission and transmission, Nano Lett. 11 (2011) 49-55.

[40] A. Serrano, O. Rodríguez De La Fuente, M.A. García, Extended and localized surface plasmons in annealed Au films on glass substrates, J. Appl. Phys. 108 (2010) 074303.

[41] G. Gupta, D. Tanaka, Y. Ito, D. Shibata, M. Shimojo, K. Furuya, et al., Absorption spectroscopy of gold nanoisland films: optical and structural characterization, Nanotechnology 20 (2009) 025703.

[42] T. Menegotto, M.B. Pereira, R.R.B. Correia, F. Horowitz, Simple modeling of plasmon resonances in Ag/SiO2 nanocomposite monolayers, Optical Society of America 50 (2010) C27-30.

\section{List of figure captions}

Figure 1 - SEM images showing the morphology evolution of Au NPs, percolating domains and films as a function of the shot number and the background gas pressure. All images are taken at the same magnification. A continuous red line separates NPs from percolating domains and thin films.

Figure 2 - SEM magnified images showing the morphology of Au NPs (a) and of percolated film (b), obtained at $1000 \mathrm{~Pa}$ at 100 and 1000 shots, respectively (a) and (b).

Figure 3 - (a) Diameter distribution of Au NPs produced at 10 Pa and 100 shots. (b) Average Au nanoparticle diameter and (c) coverage as a function of the number of shots. 
Figure 4-AFM images of Au nanoparticles deposited at $1000 \mathrm{~Pa}$ at 50 and 100 shots, respectively (a) and (b).

Figure 5 - $(a, b)$ Optical transmission spectra of Au NPs and films as a function of the shot number for a pressures equal to $10 \mathrm{~Pa}$ and $1000 \mathrm{~Pa}$, respectively. The reflectance for $10 \mathrm{~Pa}, 1000$ shots and $1000 \mathrm{~Pa}, 1000$ shots is reported respectively in (a) and (b). The inset in (b) schematically shows the extraction of FWHM. (c) Variation of the transmittance and $\lambda_{S P R}$ as a function of the number of shots at $1000 \mathrm{~Pa}$.

Figure 6-(a-c) Optical transmission spectra of Au NPs and films as a function of the background gas pressure at 50, 100 and 500 shots, respectively.

Figure $7-(a-c)$ Variation of the $\lambda_{S P R}$, the FWHM and optical transmittance at $\lambda_{S P R}$, as a function of the NPS diameter at 100 shots.

Figure 8-Variation of the $\lambda_{S P R}$ as a function of the coverage for percolated films at 500 shots.

Figure 9 - (a) Average diameter and coverage as a function of the number of shots at a fixed background gas pressure equal to $1000 \mathrm{~Pa}$. (b) The corresponding optical transmittance spectra.

Figure 10- (a) SEM images of an as deposited percolated film structure (1000 Pa, 1000 shots). (b,c) Effect of the annealing treatment at $500{ }^{\circ} \mathrm{C}$ and $650^{\circ} \mathrm{C}$, respectively $b$ and $c .(d, e)$ AFM images of Au NPs after annealing at $500{ }^{\circ} \mathrm{C}$.

Figure 11 - (a) Average Au nanoparticle diameter and coverage as a function of annealing temperature. (b) Optical transmission spectra of as-deposited Au films and after annealing treatment at $500{ }^{\circ} \mathrm{C}$ and $650{ }^{\circ} \mathrm{C}$. 


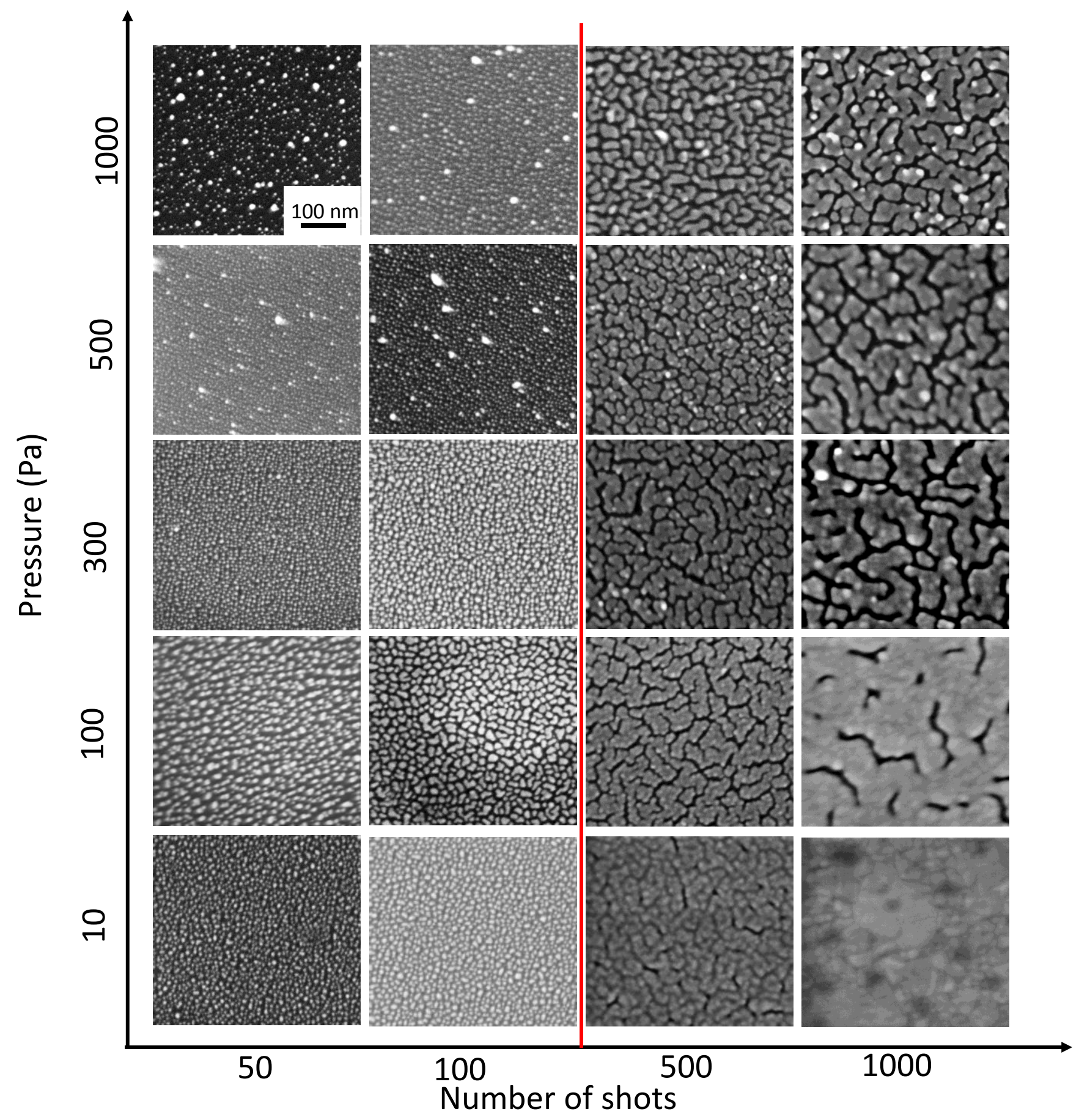

Figure 1 

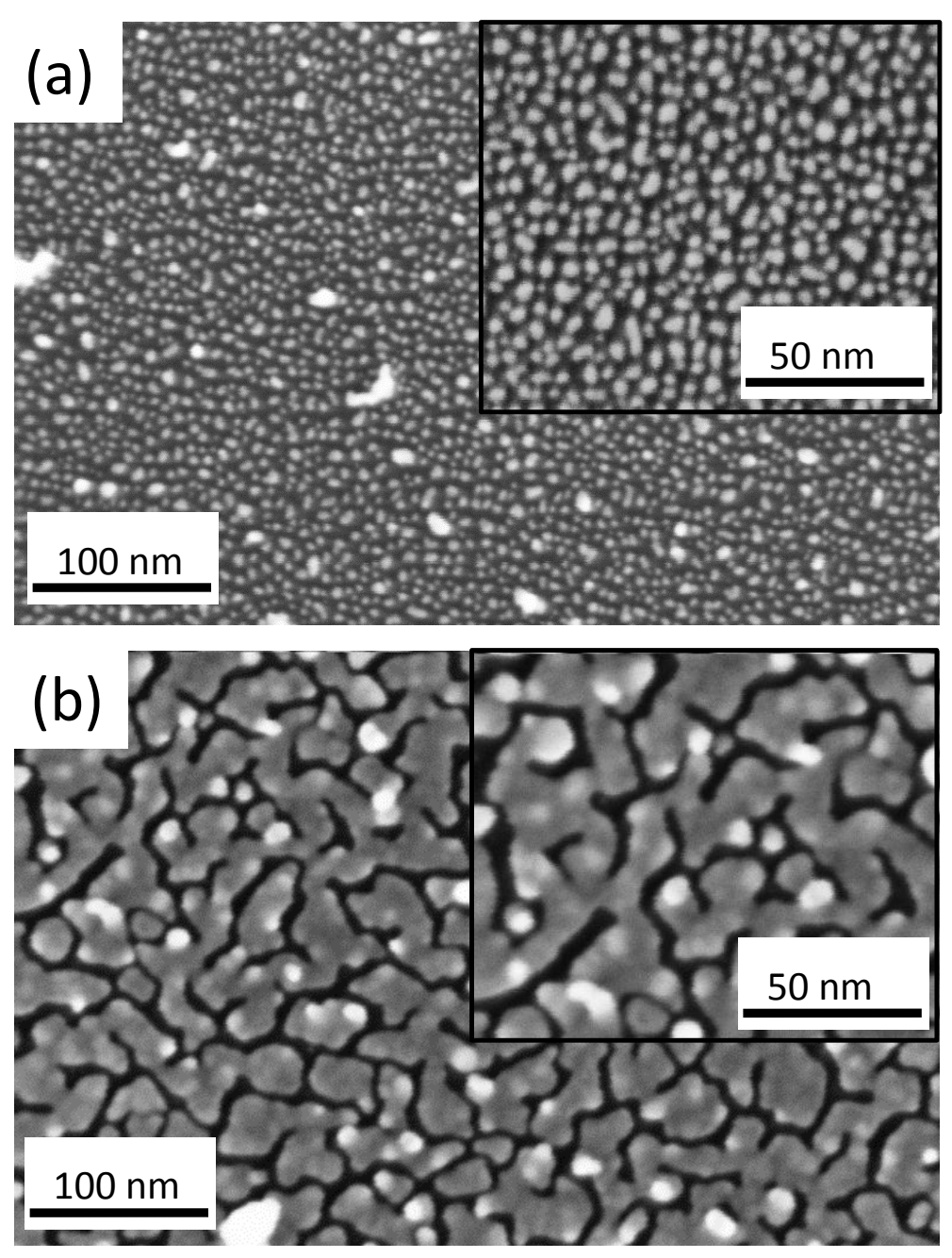

Figure 2 

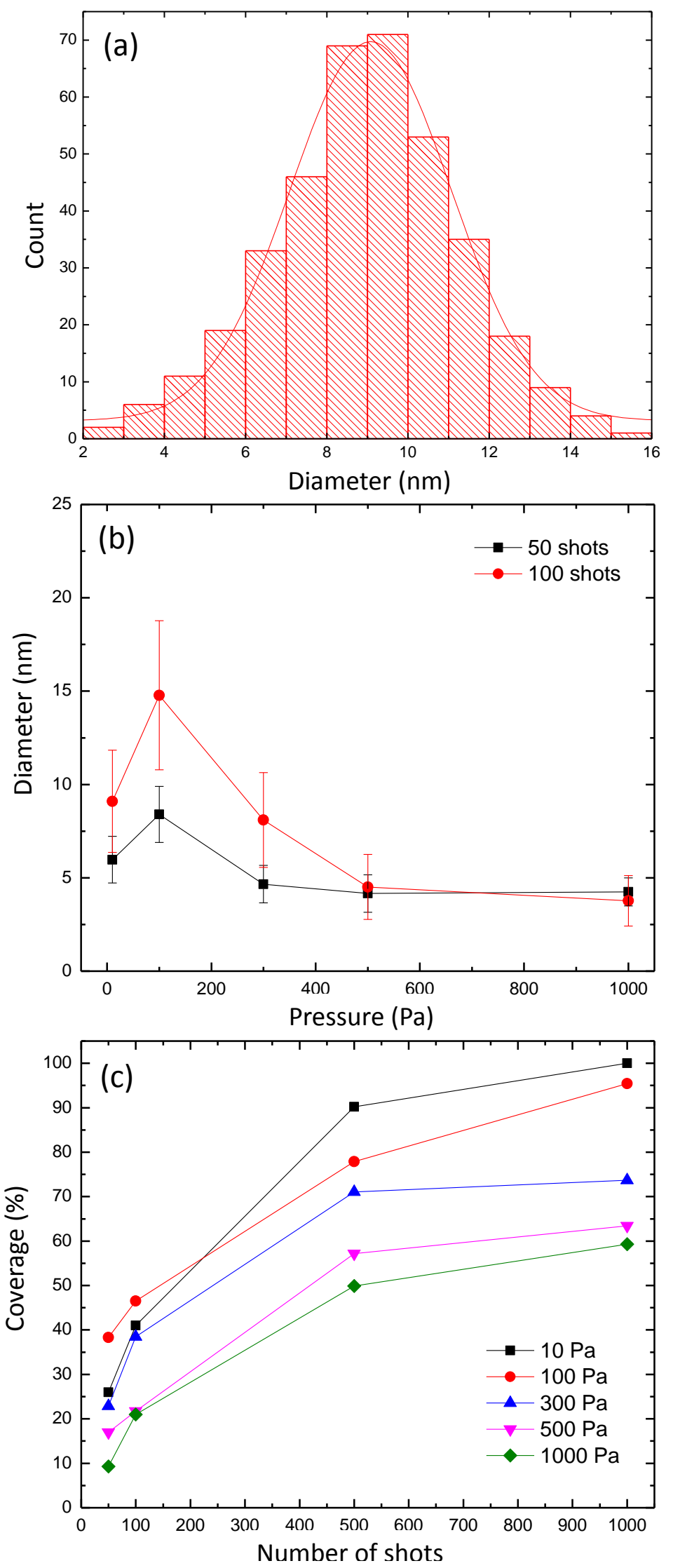

Figure 3 

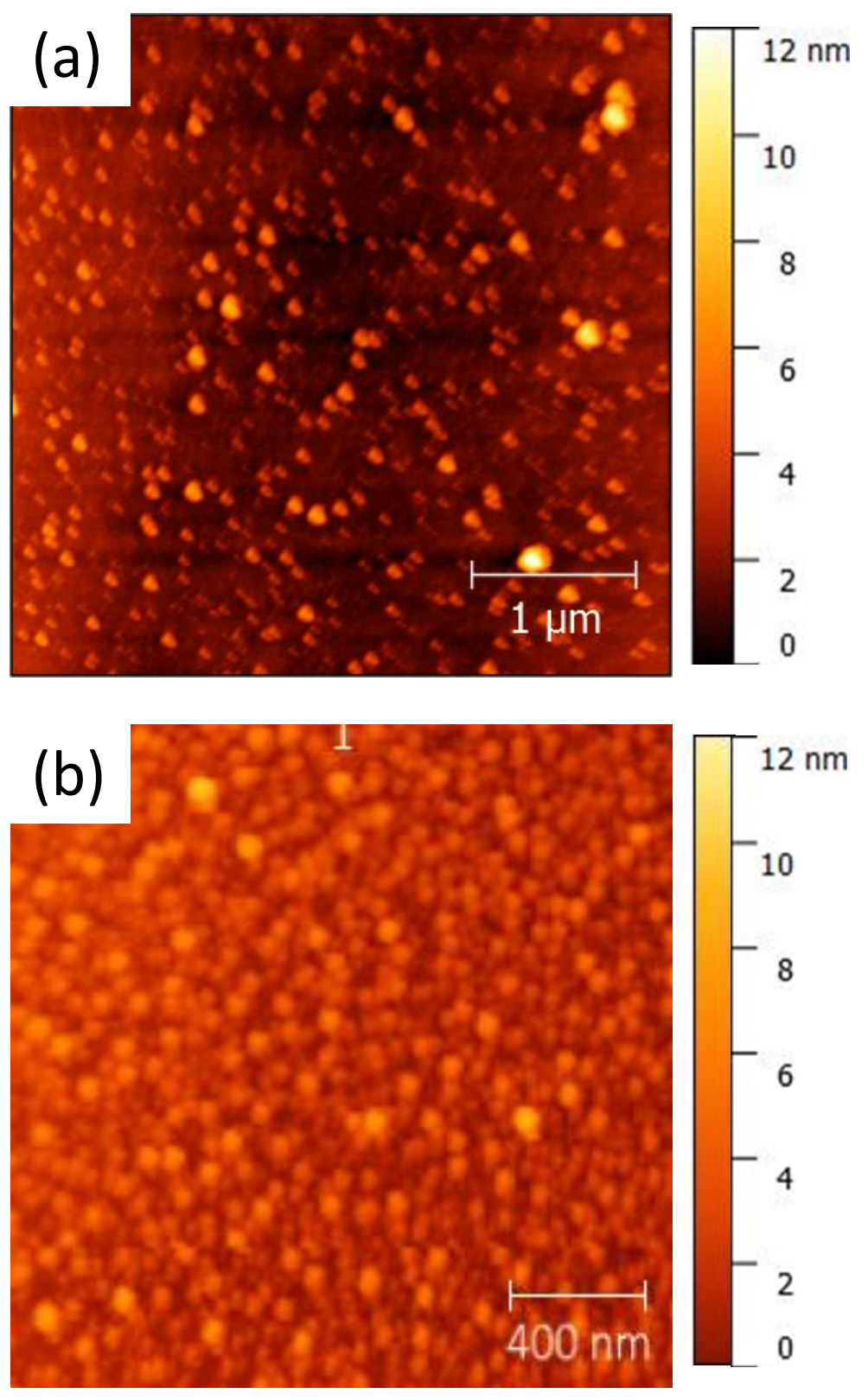

Figure 4 

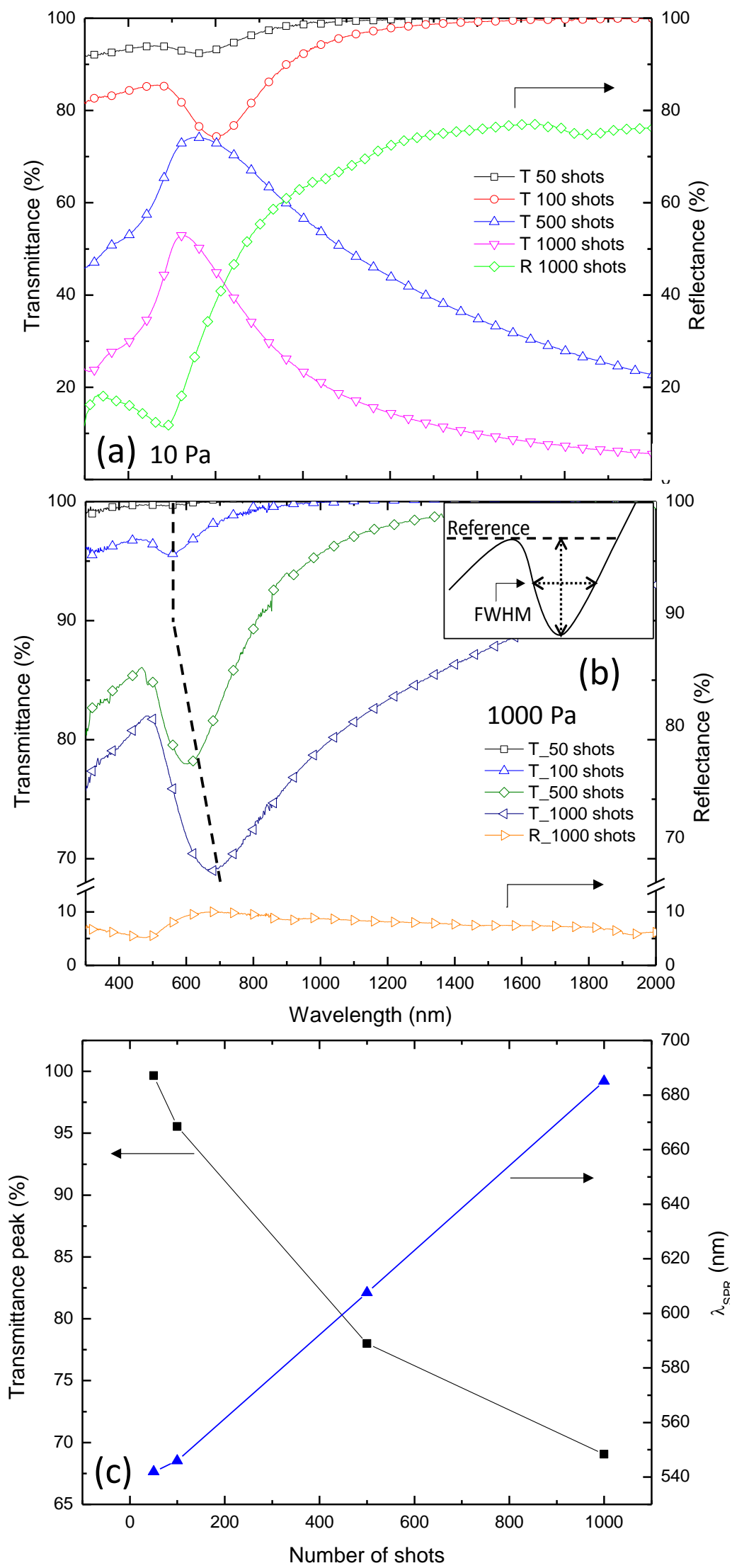

Figure 5 

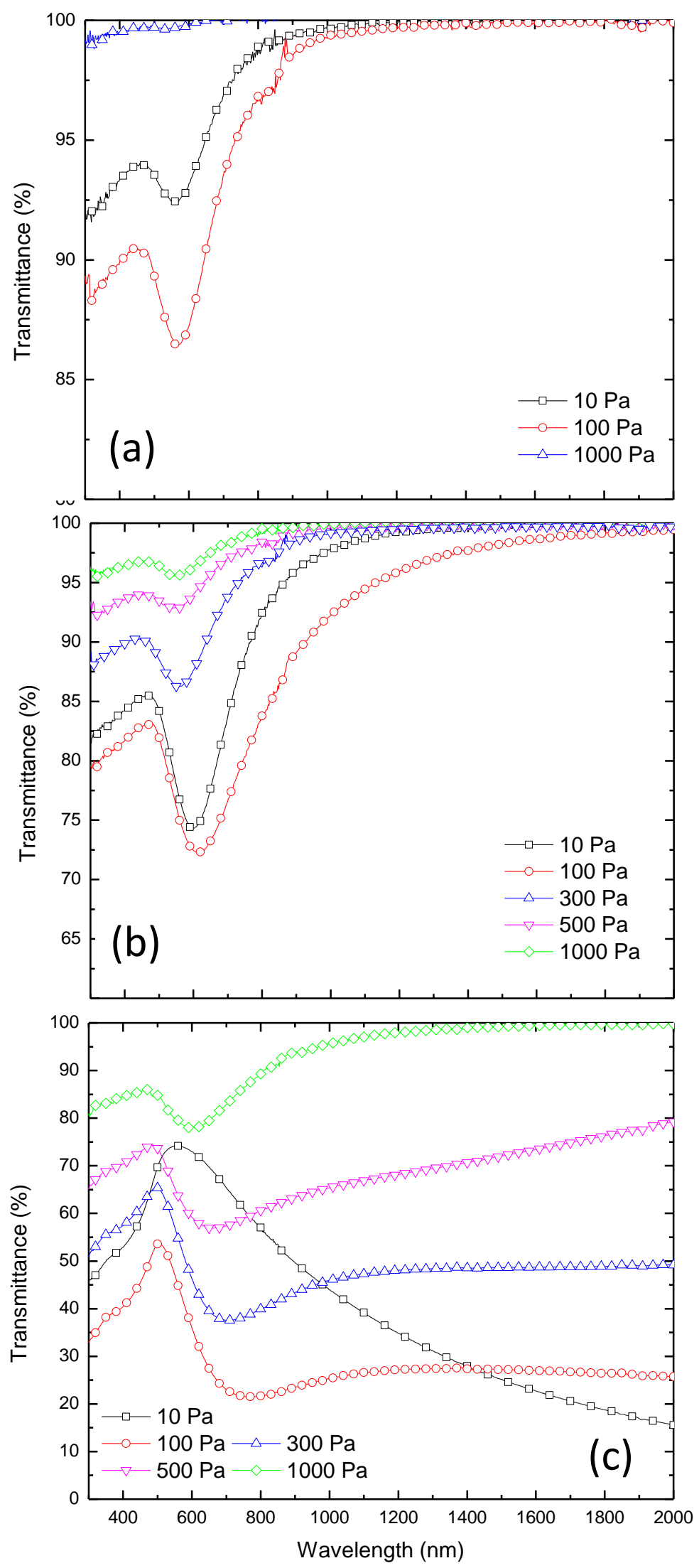

Figure 6 

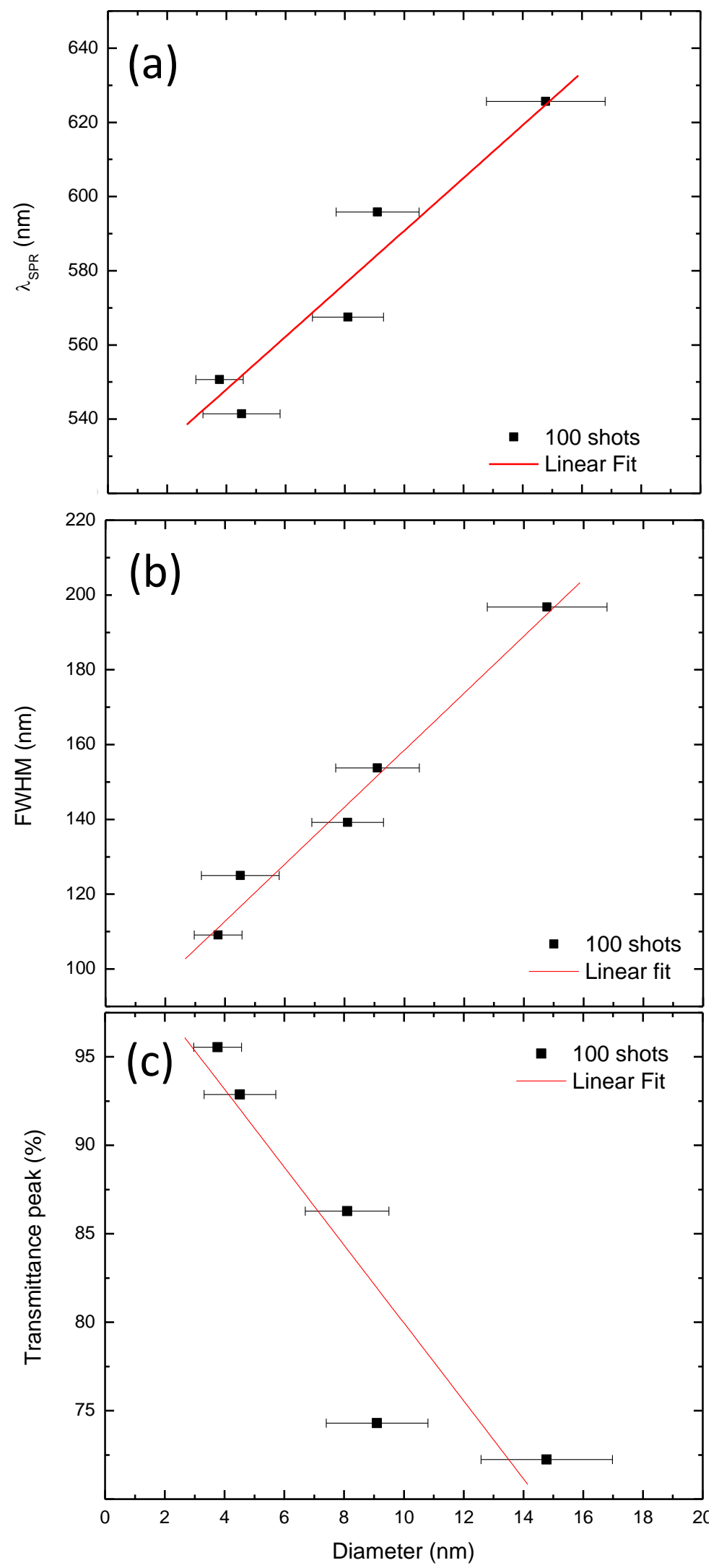

Figure 7 


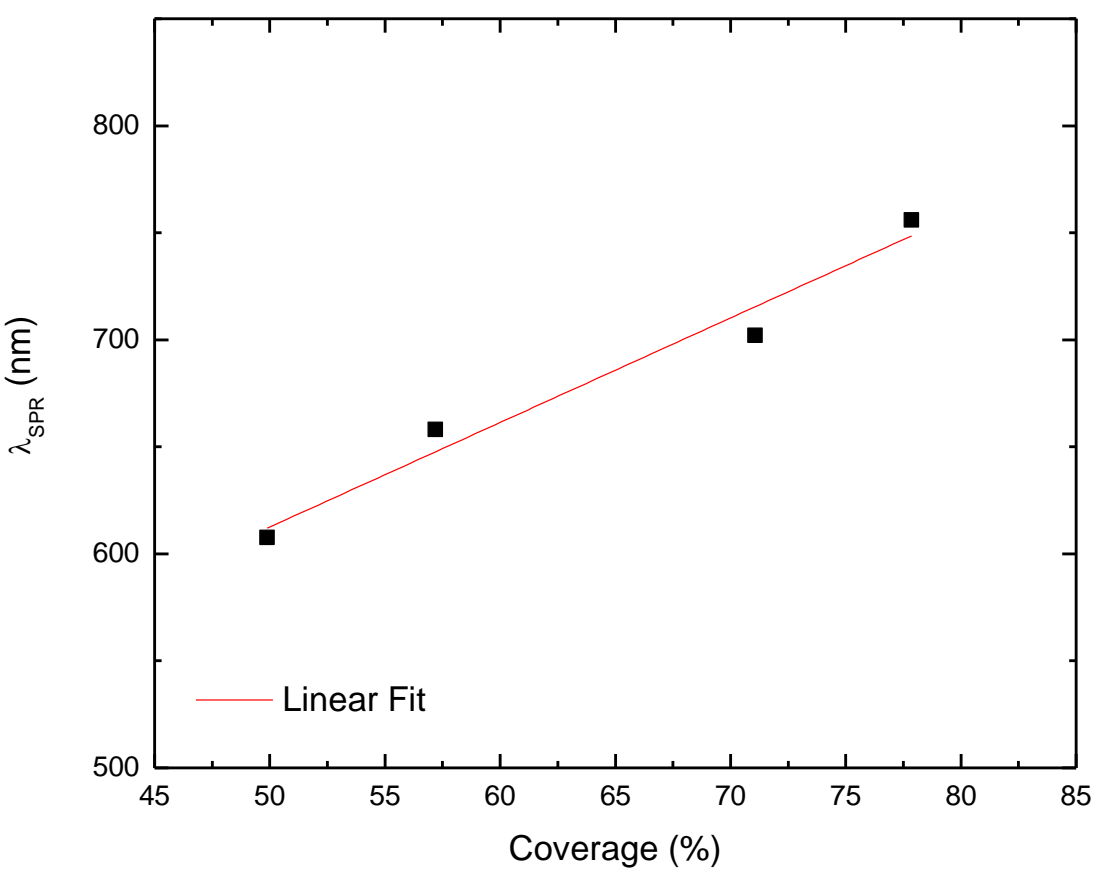

Figure 8 

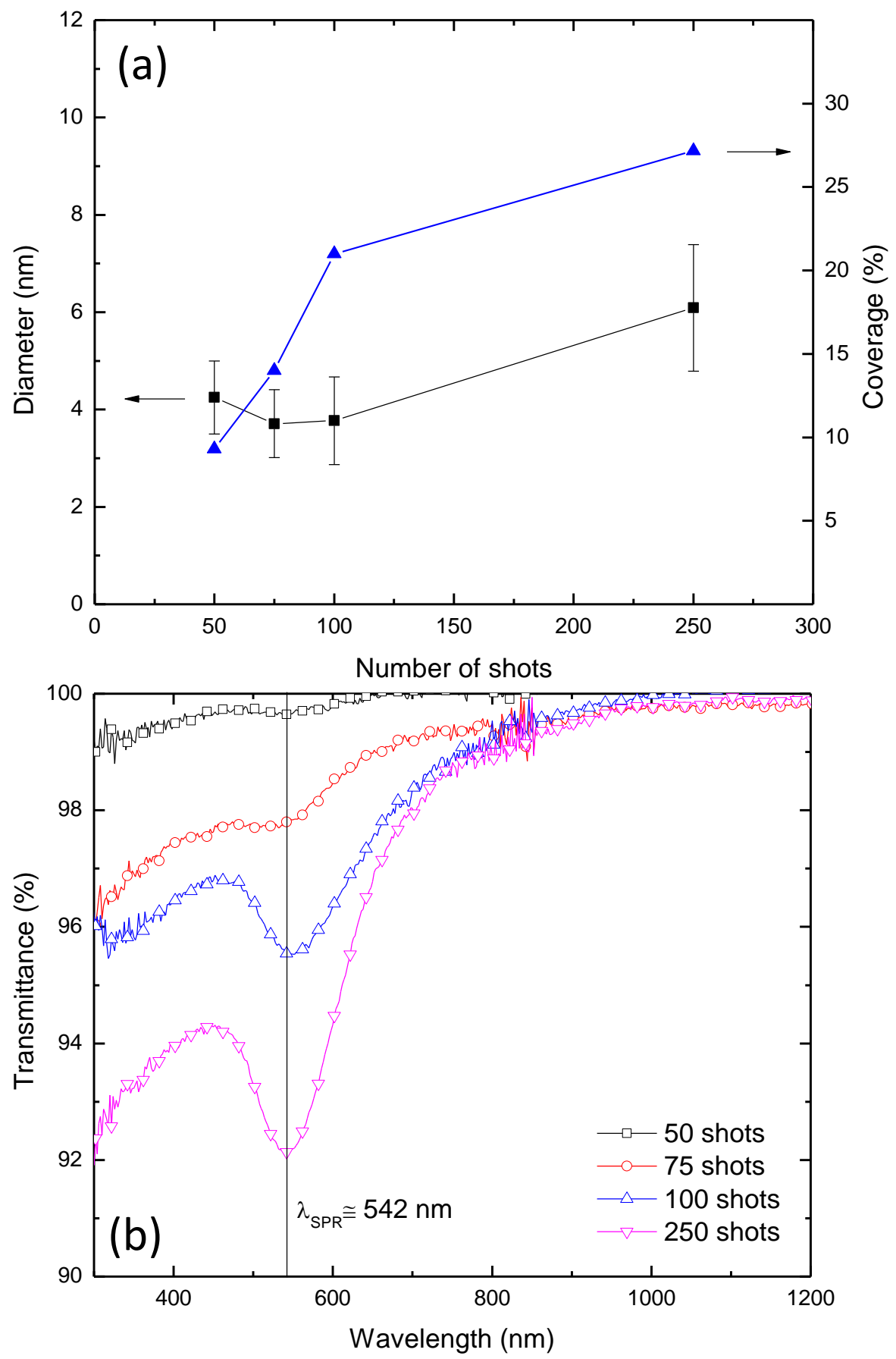

Figure 9 

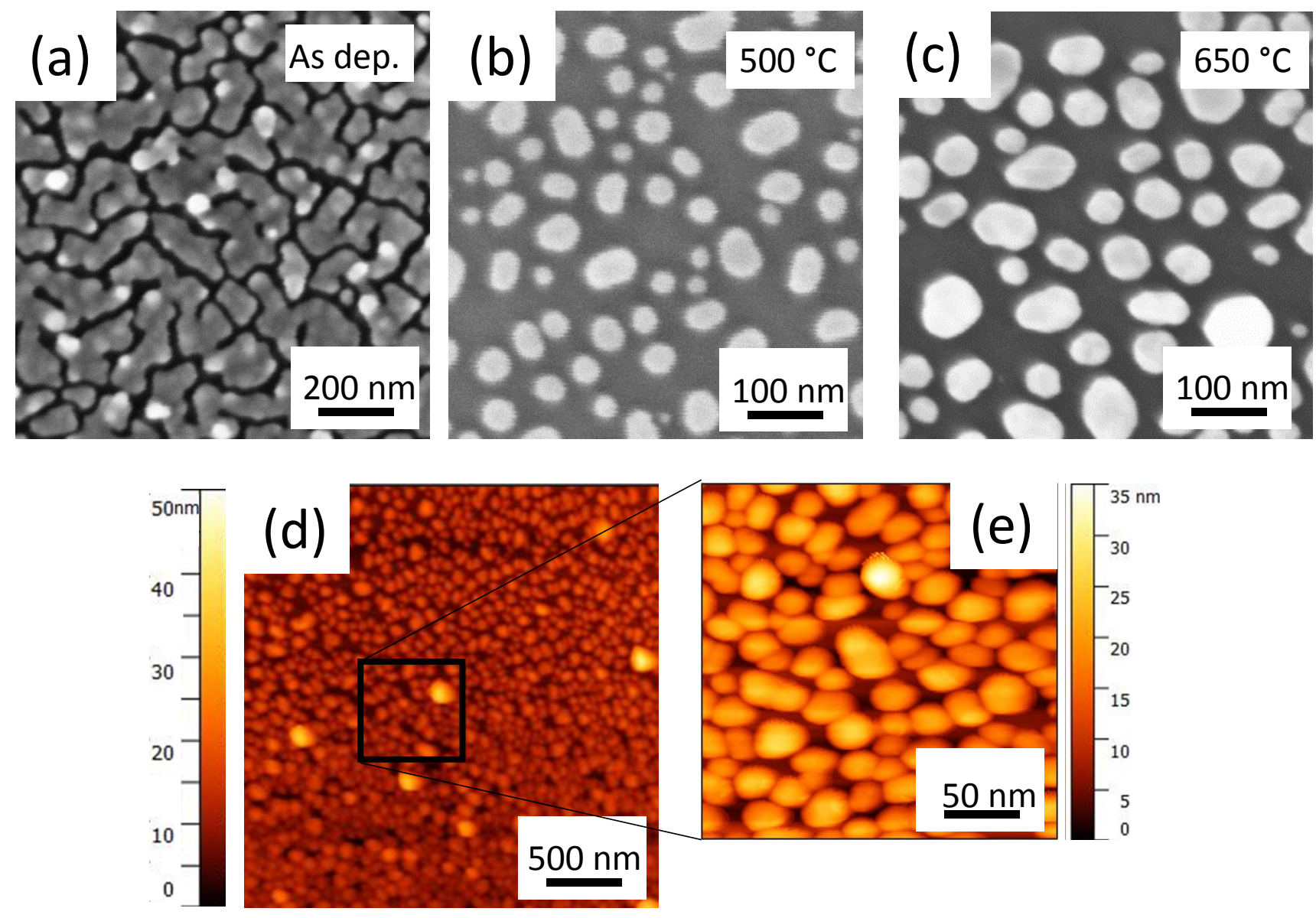

Figure 10 

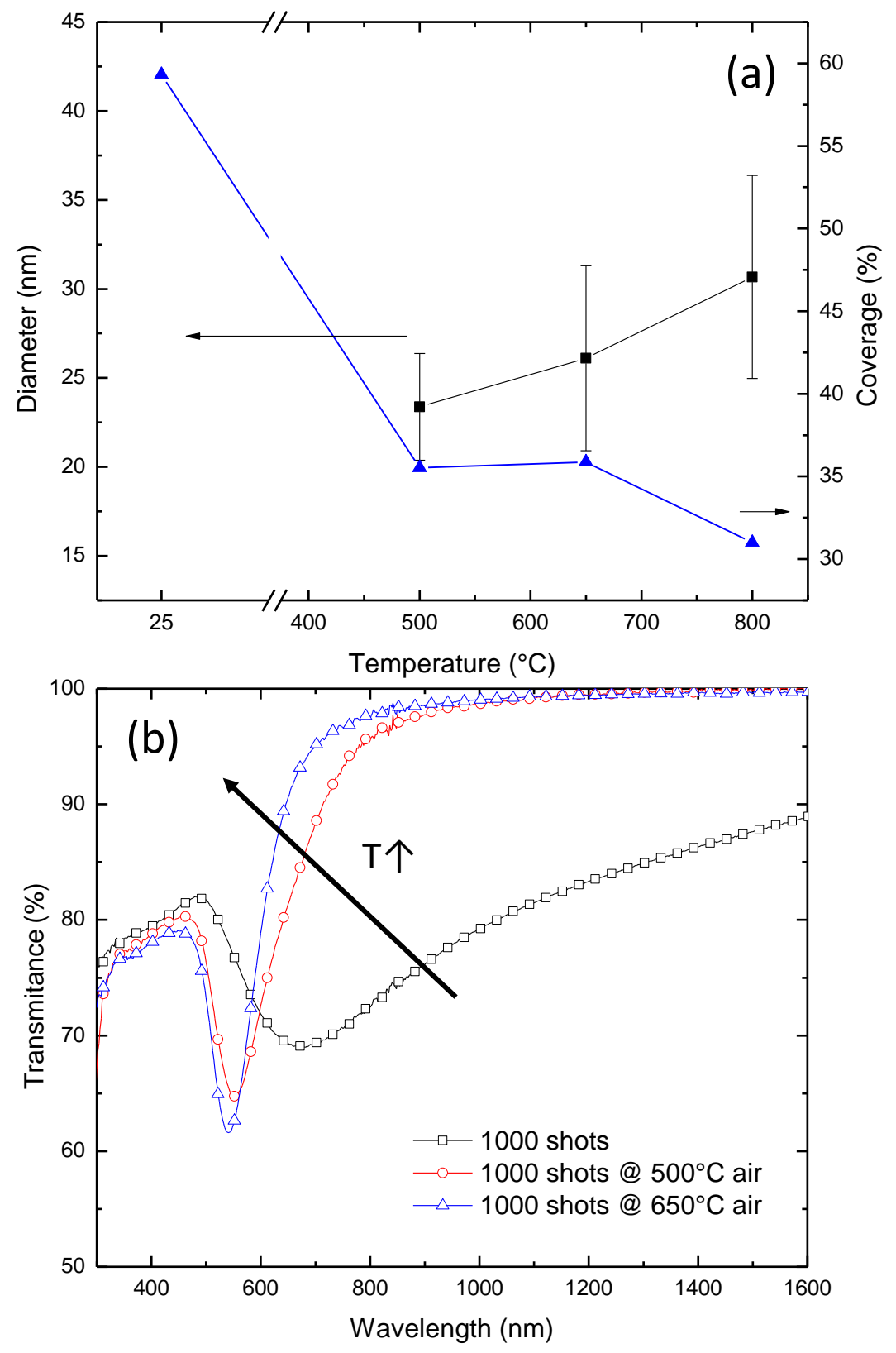

Figure 11 